JOURNAL OF THE

AMERICAN MATHEMATICAL SOCIETY

Volume 19, Number 2, Pages 345-355

S 0894-0347(05)00509-6

Article electronically published on December 2, 2005

\title{
GLOBAL $F$-REGULARITY OF SCHUBERT VARIETIES WITH APPLICATIONS TO D-MODULES
}

\author{
NIELS LAURITZEN, ULF RABEN-PEDERSEN, AND JESPER FUNCH THOMSEN
}

A projective algebraic variety $X$ over an algebraically closed field $k$ of positive characteristic is called globally $F$-regular if the section $\operatorname{ring} S(\mathcal{L})=\bigoplus_{n>0} \mathrm{H}^{0}\left(X, \mathcal{L}^{n}\right)$ of an ample line bundle $\mathcal{L}$ on $X$ is strongly $F$-regular in the sense of Hochster and Huneke 9] (cf. Definition 1.1). This important notion was introduced by Karen Smith in [18.

In this paper we prove that Schubert varieties are globally $F$-regular. An immediate consequence is that local rings of Schubert varieties are strongly $F$-regular and thereby $F$-rational. Another consequence is that local rings of varieties (like determinantal varieties) that can be identified with open subsets of Schubert varieties (cf. [13]) are strongly $F$-regular

Let $X$ denote a flag variety and $Y \subset X$ a Schubert variety over $k$. Then the local cohomology sheaves $\mathcal{H}_{Y}^{j}\left(\mathcal{O}_{X}\right)$ are equivariant (for the action of the Borel subgroup) and holonomic (in the sense of [4]) $\mathcal{D}_{X}$-modules. As an application of $F$ rationality of Schubert varieties we apply recent results of Blickle (cf. 2]) to prove that the simple objects in the category of equivariant and holonomic $\mathcal{D}_{X}$-modules are precisely the local cohomology sheaves $\mathcal{H}_{Y}^{c}\left(\mathcal{O}_{X}\right)$, where $c$ is the codimension of $Y$ in $X$. Using a local Grothendieck-Cousin complex from [11, we prove that the decomposition of the local cohomology modules with support in Bruhat cells is multiplicity free (see 4.2 ).

In characteristic zero the local cohomology modules with support in Bruhat cells correspond to dual Verma modules. In this setting the decomposition behavior and the simple $\mathcal{D}_{X}$-modules arise from intersection cohomology complexes of Schubert varieties by the Riemann-Hilbert correspondence. If we pick the singular codimension one Schubert variety $Y$ in the full flag variety $Z$ for $\mathrm{SL}_{4}$ in characteristic zero, computations in Kazhdan-Lusztig theory show that $\mathcal{H}_{Y}^{1}\left(\mathcal{O}_{Z}\right)$ is not a simple $\mathcal{D}_{Z}$-module (see 4 .1.1).

\section{Global F-Regularity and Frobenius splitting}

Let $k$ denote a field of characteristic $p>0$ and $R$ a finitely generated $k$-algebra. For an $R$-module $M$ we define $F_{*}^{e} M$ to be the $R$-module which is equal to $M$ as an abelian group with $R$-action given by $r \cdot m=r^{p^{e}} m$.

Definition 1.1 (9). The ring $R$ is said to be strongly $F$-regular if for every $c \in R$, not contained in any minimal prime of $R$, there exists a positive integer $e \geq 0$ such

Received by the editors February 18, 2004.

2000 Mathematics Subject Classification. Primary 32C38, 14B15.

(C)2005 American Mathematical Society

Reverts to public domain 28 years from publication 
that the map of $R$-modules

$$
\begin{gathered}
R \rightarrow F_{*}^{e} R, \\
1 \mapsto c
\end{gathered}
$$

is split.

The concept of strong $F$-regularity has been extended to projective varieties over $k$ in the following way.

Definition 1.2 ([18]). The projective variety $X$ is said to be globally F-regular if there exists an ample line bundle $\mathcal{L}$ on $X$ such that the section ring

$$
S(\mathcal{L})=\bigoplus_{n \in \mathbb{N}} \mathrm{H}^{0}\left(X, \mathcal{L}^{n}\right)
$$

is strongly $F$-regular.

In case $X$ is globally $F$-regular, it can be shown that the section ring of any ample line bundle is strongly $F$-regular. Moreover, all the local rings $\mathcal{O}_{X, x}$, at points $x$ of $X$, can be shown to be strongly $F$-regular (see [18]).

Let $X$ be a variety over $k$. The absolute Frobenius morphism on $X$ is the morphism $F: X \rightarrow X$ of schemes, which is the identity on the set of points and where the associated map of sheaves

$$
F^{\sharp}: \mathcal{O}_{X} \rightarrow F_{*} \mathcal{O}_{X}
$$

is the $p$-th power map.

Let $D$ be an effective Cartier divisor on $X$ and let $s$ denote the associated section of the line bundle $\mathcal{O}_{X}(D)$. We define $X$ to be Frobenius split along $D$ if the map of $\mathcal{O}_{X}$-modules

$$
\begin{gathered}
\mathcal{O}_{X} \rightarrow F_{*} \mathcal{O}_{X}(D), \\
1 \rightarrow s
\end{gathered}
$$

splits. When $D=0$, we also say that $X$ is Frobenius split [15]. Moreover, if there exists a positive integer $e$ such that the map of $\mathcal{O}_{X}$-modules

$$
\begin{gathered}
\mathcal{O}_{X} \rightarrow F_{*}^{e} \mathcal{O}_{X}(D), \\
1 \rightarrow s
\end{gathered}
$$

splits, then we say that $X$ is stably Frobenius split along $D[18$.

Proposition 1.3. Let $X$ denote a variety and let $D$ and $D^{\prime}$ be effective divisors on $X$. If $X$ is stably Frobenius split along $D$, then $X$ is stably Frobenius split along $p D$. Moreover, if $D^{\prime} \leq D$ and $X$ is stably Frobenius split along $D$, then $X$ is also stably Frobenius split along $D^{\prime}$.

Proof. See Section 3 in [18.

Consider now the situation when $X$ is Frobenius split (along the zero divisor), and let $Y$ denote a closed subscheme of $X$ with sheaf of ideals $\mathcal{I}_{Y}$. Let $\phi: F_{*} \mathcal{O}_{X} \rightarrow$ $\mathcal{O}_{X}$ denote the Frobenius splitting. We then say that $Y$ is compatibly Frobenius split if $\phi\left(F_{*} \mathcal{I}_{Y}\right) \subseteq \mathcal{I}_{Y}$. When $Y$ is an effective Cartier divisor on $X$, this concept relates to Frobenius splitting along $Y$ in the following way.

Lemma 1.1. Let $Y$ denote an effective Cartier divisor on $X$. If $Y$ is compatibly Frobenius split in $X$, then $X$ is Frobenius split along $(p-1) Y$. 
Proof. Assume that $X$ is Frobenius split and let $\phi: F_{*} \mathcal{O}_{X} \rightarrow \mathcal{O}_{X}$ denote the associated splitting. The ideal sheaf of $Y$ is isomorphic to $\mathcal{O}_{X}(-Y)$ and the inclusion $\mathcal{O}_{X}(-Y) \simeq \mathcal{I}_{Y} \subseteq \mathcal{O}_{X}$ is given by multiplication with the section $s \in \mathcal{O}_{X}(Y)$ defining $Y$. If $Y$ is compatibly split, then the $p$-power map

$$
\mathcal{O}_{X}(-Y) \simeq \mathcal{I}_{Y} \rightarrow F_{*} \mathcal{I}_{Y} \simeq F_{*} \mathcal{O}_{X}(-Y)
$$

splits. Tensoring this map with $\mathcal{O}_{X}(Y)$ induces a split map

$$
\begin{aligned}
\mathcal{O}_{X} \rightarrow\left(F_{*} \mathcal{O}_{X}(-Y)\right) & \otimes \mathcal{O}_{X}(Y) \simeq F_{*} \mathcal{O}_{X}((p-1) Y), \\
1 & \mapsto s^{p-1}
\end{aligned}
$$

where the isomorphism on the left follows from the projection formula and the fact that $F^{*} \mathcal{L} \simeq \mathcal{L}^{\otimes p}$ for every line bundle $\mathcal{L}$ on $X$. This completes the proof.

For a projective variety $X$, global $F$-regularity is equivalent to $X$ being stably Frobenius split along every effective Cartier divisor (cf. Theorem 3.10(c) in [18]). This leads to the following result.

Lemma 1.2. Let $\pi: X \rightarrow Y$ be a morphism of projective varieties over $k$ satisfying $\pi_{*} \mathcal{O}_{X}=\mathcal{O}_{Y}$. If $X$ is globally $F$-regular, then $Y$ is also globally $F$-regular. In particular, if $\pi$ is birational, $X$ is globally F-regular, and $Y$ is normal, then $Y$ is globally F-regular.

Proof. Let $D$ denote an effective Cartier divisor on $Y$, and let $s$ denote the corresponding section of the associated line bundle $\mathcal{O}_{Y}(D)$. The pullback of $D$ to $X$ will be denoted by $D^{\prime}$, and the associated section of $\mathcal{O}_{X}\left(D^{\prime}\right)$ is denoted by $s^{\prime}$. Hence, assuming that $X$ is globally $F$-regular, it follows, by Theorem 3.10 in [18, that there exists an integer $e$ such that the morphism of $\mathcal{O}_{X}$-modules

$$
\begin{gathered}
\mathcal{O}_{X} \rightarrow F_{*}^{e} \mathcal{O}_{X}\left(D^{\prime}\right), \\
1 \mapsto s^{\prime}
\end{gathered}
$$

splits. Applying the functor $\pi_{*}$ to this split morphism and the assumption $\pi_{*} \mathcal{O}_{X}=$ $\mathcal{O}_{Y}$, we conclude that the morphism

$$
\begin{gathered}
\mathcal{O}_{Y} \rightarrow F_{*}^{e} \mathcal{O}_{Y}(D), \\
1 \mapsto s
\end{gathered}
$$

of $\mathcal{O}_{Y}$-modules splits. The global $F$-regularity of $Y$ now follows from Theorem 3.10 in [18]. The "in particular" statement follows as $\pi_{*} \mathcal{O}_{X}=\mathcal{O}_{Y}$ if $\pi$ is birational and $Y$ is normal.

\section{Schubert VARIETies are Globally $F$-REgular}

Let $G$ be a connected and simply connected semisimple linear algebraic group $G$ over $k$. Let $B \subset G$ denote a fixed Borel subgroup, $T$ a maximal torus in $B$, and $P$ a parabolic subgroup containing $B$. The $B$-action on the flag variety $G / P$ has finitely many orbits $C(w)$ parametrized by left cosets $w \in W / W_{P}$ in the Weyl group $W$ of $G$ with respect to the Weyl group $W_{P}$ of $P$. We let $X(w)$ denote the closure of $C(w)$ in $G / P$. This is the Schubert variety corresponding to $w$. The Weyl group comes with a natural partial order (the Bruhat order) given by $v \leq w$ if and only if $X(v) \subseteq X(w)$ for $v, w \in W$. We let $\ell(w)$ denote the length of the Weyl group element $w \in W$. For details on the theory of linear algebraic groups we refer to [19]. 
2.1. Bott-Samelson varieties. If $s \in W$ is a simple reflection, the Schubert variety $X(s)$ coincides with the variety $P_{s} / B \simeq \mathbb{P}^{1} \subset G / B$ for the minimal parabolic subgroup $P_{s}=B \cup B s B$.

Let $w=\left(s_{1}, s_{2}, \ldots, s_{l}\right)$ denote a collection of simple reflections in $W$ and let $P_{i}$ denote the minimal parabolic subgroup associated with $s_{i}$. The product

$$
P_{w}=P_{1} \times P_{2} \times \cdots \times P_{l}
$$

comes with a right action of $B^{l}$ defined as

$$
\left(p_{1}, p_{2}, \cdots, p_{l}\right)\left(b_{1}, b_{2}, \cdots, b_{l}\right)=\left(p_{1} b_{1}, b_{1}^{-1} p_{2} b_{2}, \cdots, b_{l-1}^{-1} p_{l} b_{l}\right) .
$$

The quotient $Z(w)=P_{w} / B^{l}$ is a smooth projective variety of dimension $l$ called a Bott-Samelson variety.

Fix an integer $1 \leq i \leq l$ and consider the closed set of points $Z_{i}$ in $Z\left(\left(s_{1}, \ldots, s_{l}\right)\right)$ which may be represented by a point in $P_{1} \times P_{2} \times \cdots \times P_{l}$ of the form $\left(p_{1}, \ldots, p_{l}\right)$ with $p_{i}$ equal to the identity element in $G$. Then $Z_{i}$ is an irreducible closed subvariety of $Z\left(s_{1}, \ldots, s_{l}\right)$ isomorphic to the Bott-Samelson variety $Z\left(s_{1}, \ldots, \hat{s_{i}}, \ldots, s_{l}\right)$. For further details and references on Bott-Samelson varieties we refer the reader to [14].

Proposition 2.1. The Bott-Samelson variety $Z=Z(w)$ is globally F-regular.

Proof. As $Z$ is smooth, it is enough to prove that $Z$ is stably Frobenius split along an ample divisor $D$ (see Theorem $3.10[18]$ ). By Theorem 1 in [15] there exists a Frobenius splitting of $Z$ compatibly splitting the effective divisor $\sum_{i} Z_{i}$. (Strictly speaking, this result in 15] only deals with the case where $s_{1} \cdots s_{l}$ is a reduced expression. However, the general case follows in exactly the same manner.)

By Lemma 1.1 and Proposition 1.3 this implies that $X$ is stably Frobenius split along any divisor of the form $\sum_{i} m_{i} Z_{i}, m_{i} \in \mathbb{N}$. But by Lemma 6.1 in [14] there exist integers $m_{i}>0$ such that the divisor $\sum_{i} m_{i} Z_{i}$ is ample. This completes the proof.

2.2. Global $F$-regularity of Schubert varieties. Consider a Schubert variety $X(w)$ in $G / B$ corresponding to an element $w$ in the Weyl group $W$. Write $w$ as a product of simple reflections

$$
w=s_{1} s_{2} \cdots s_{l},
$$

with $l$ minimal (i.e., $l=\ell(w)$ the length of $w$ ). By the Bruhat decomposition the morphism

$$
\begin{gathered}
P_{1} \times P_{2} \times \cdots \times P_{l} \rightarrow X(w), \\
\left(p_{1}, p_{2}, \cdots, p_{l}\right) \mapsto p_{1} p_{2} \cdots p_{l} B
\end{gathered}
$$

induces a birational morphism

$$
Z\left(\left(s_{1}, \ldots, s_{l}\right)\right) \rightarrow X(w)
$$

This leads to the following result.

Theorem 2.2. Let $P$ denote a parabolic subgroup in $G$ which contains $B$. A Schubert variety $X$ in $G / P$ is globally F-regular. 
Proof. It is well known that Schubert varieties are normal. The case $P=B$ follows immediately from Lemma 1.2 and Proposition 2.1, together with the existence of the birational map

$$
Z\left(s_{1}, \ldots, s_{l}\right) \rightarrow X(w),
$$

described above.

Consider now a general parabolic subgroup $P$. The inverse image of $X$, by the canonical map $\pi: G / B \rightarrow G / P$, is then a Schubert variety $X(w)$ in $G / B$ for some $w \in W$. Choose $w^{\prime}$ to be a minimal length representative for the left coset $w W_{P}$ in $W / W_{P}$. By the Bruhat decomposition it follows that the induced map

$$
\pi: X\left(w^{\prime}\right) \rightarrow X
$$

is birational. As $X\left(w^{\prime}\right)$ is globally $F$-regular, the statement is now a consequence of Lemma 1.2.

\section{3. $F$-Rationality and $\mathcal{D}$-MOdUles}

Let $R$ denote a commutative algebra over a perfect field $k$. The ring of $k$-linear differential operator $D_{k}(R)$ on $R$ is an $R \otimes_{k} R$-subalgebra of $\operatorname{End}_{k}(R)$ defined by

$$
D_{k}(R)=\left\{\phi \in \operatorname{End}_{k}(R): I^{n} \cdot \phi=0, n \gg 0\right\},
$$

where $I$ denotes the kernel of the product map $R \otimes_{k} R \rightarrow R$. The $R \otimes_{k} R$-submodules

$$
D_{k}^{n}(R)=\left\{\phi \in \operatorname{End}_{k}(R): I^{n+1} \cdot \phi=0\right\}
$$

define a filtration of $D_{k}(R)$. Elements in $D_{k}^{n}(R)$ are called differential operators of degree $\leq n$. When $I$ is a finitely generated ideal, there is a second filtration of $D_{k}(R)$ given by the $R \otimes_{k} R$-submodules

$$
D_{k}^{(n)}(R)=\left\{\phi \in \operatorname{End}_{k}(R): I^{(n+1)} \cdot \phi=0\right\},
$$

where $I^{(n)}$ denotes the ideal in $R \otimes_{k} R$ generated by elements of the form $a^{n}, a \in I$. This filtration is particularly nice when the characteristic $p$ of $k$ is positive. In this case $I^{\left(p^{n}\right)}$ is generated by elements of the form $a^{p^{n}} \otimes 1-1 \otimes a^{p^{n}}$, and hence $D_{k}^{\left(p^{n}-1\right)}(R)=\operatorname{End}_{R^{p^{n}}}(R)$, where $R^{p^{n}}$ denotes the subring of $R$ of $p^{n}$-powers (here we use that $k$ is algebraically closed and hence perfect). In particular,

$$
D_{k}(R)=\bigcup_{n} \operatorname{End}_{R^{p^{n}}}(R) .
$$

The right side of this equation shows that $D_{k}(R)$ is independent of $k$, and we therefore suppress $k$ from the notation and write $D(R)$ instead of $D_{k}(R)$.

Lemma 3.1. Assume that $k$ has positive characteristic $p$ and that $R$ is a finitely generated k-algebra. For every multiplicative subset $S$ of $R$ there exists a natural isomorphism of left $R_{S}$-modules

$$
(D(R))_{S} \simeq D\left(R_{S}\right)
$$

where the localization on the left is performed as a left $R$-module.

Proof. Fix a positive integer $n$. As $R$ is a finitely generated $k$-algebra, it is finitely generated as a module over the subring $R^{p^{n}}$. This implies that the exists a natural isomorphism

$$
\operatorname{End}_{R^{p^{n}}}(R)_{S} \simeq \operatorname{End}_{R_{S}^{p^{n}}}\left(R_{S}\right)
$$


Now conclude the argument by using the description of $D(R)$ above (in positive characteristic).

3.1. Sheaves of differential operators. Let $X$ be a variety over $k$. The sheaf of $k$-linear differential operators $\mathcal{D}_{X}$ on $X$ (cf. 6, $\left.\$ 16.8\right)$ is an $\mathcal{O}_{X}$-bisubalgebra of $\mathcal{E} n d_{k}\left(\mathcal{O}_{X}\right)$ which is quasicoherent for both $\mathcal{O}_{X}$-module structures. When $X=$ $\operatorname{Spec}(R)$ is affine, the sheaf $\mathcal{D}_{X}$ coincides with the quasicoherent $\mathcal{O}_{X}$-bialgebra associated to the $R$-bialgebra $D_{k}(R)$ defined above.

Lemma 3.2. Assume that $k$ has positive characteristic. For every $x \in X$ the natural morphism of algebras

$$
\phi:\left(\mathcal{D}_{X}\right)_{x} \rightarrow D\left(\mathcal{O}_{X, x}\right)
$$

is an isomorphism of $\mathcal{O}_{X, x}$-bimodules.

Proof. We may assume that $X=\operatorname{Spec}(R)$ is affine. When $\mathfrak{p}$ denotes the prime ideal in $R$ associated with $x$, we may identify $\mathcal{O}_{X, x}$ with $R_{\mathfrak{p}}$ and $\left(\mathcal{D}_{X}\right)_{x}$ with $D(R)_{\mathfrak{p}}$ (as a left $\mathcal{O}_{X, x}$-bimodule). The result now follows from Lemma 3.1 above.

3.2. Local cohomology and $\mathcal{D}$-modules. A sheaf of abelian groups $\mathcal{F}$ on a variety $X$ is called a $\mathcal{D}_{X}$-module if $\mathcal{F}$ is a module over the sheaf of algebras $\mathcal{D}_{X}$ such that the $\mathcal{O}_{X}$-structure, induced by the inclusion of $\mathcal{O}_{X}$ in $\mathcal{D}_{X}$, is quasicoherent.

For a locally closed subset $C$ of $X$ and a sheaf $\mathcal{F}$ of abelian groups on $X$, we let $\mathcal{H}_{C}^{i}(\mathcal{F})$ denote the $i$-th local cohomology sheaf with support in $C$ (cf. [7]). If $\mathcal{F}$ is a $\mathcal{D}_{X}$-module, then $\mathcal{H}_{C}^{i}(\mathcal{F})$ is a $\mathcal{D}_{X}$-module for any locally closed subset $C \subseteq X$. In particular, we may regard $\mathcal{H}_{C}^{i}\left(\mathcal{O}_{X}\right)$ as a $\mathcal{D}_{X}$-module.

Lemma 3.3. Let $C$ be a locally closed subset of a smooth variety $X$. Then the $\mathcal{D}_{X}$-module $\mathcal{H}_{C}^{i}\left(\mathcal{O}_{X}\right)$ has finite length.

Proof. The proof depends on the concept of filtration holonomicity defined by Bögvad in [3]. By Prop. 3.7. in [3] the $\mathcal{D}_{X}$-module $\mathcal{H}_{C}^{i}\left(\mathcal{O}_{X}\right)$ is filtration holonomic. But any filtration holonomic module has finite length as a $\mathcal{D}_{X}$-module (Prop. 3.2. in [3]).

3.3. Support of finite length $\mathcal{D}$-modules. Let $X$ be a variety over $k$. In this section we prove that the support of any $\mathcal{D}_{X}$-module of finite length is closed.

Lemma 3.4. Let $X$ be a variety over $k$ and let $\mathcal{F}$ denote a simple $\mathcal{D}_{X}$-module. Then $\mathcal{F}_{\mid U}$ is a simple $\mathcal{D}_{U}$-module for any open subset $U$ of $X$.

Proof. Let $i: U \rightarrow X$ denote the inclusion map. The restriction map $\mathcal{F} \rightarrow i_{*} \mathcal{F}_{\mid U}$ is a map of $\mathcal{D}_{X}$-modules. Let $\mathcal{M}$ denote a $\mathcal{D}_{U}$-submodule of $\mathcal{F}_{\mid U}$ and consider the composed map

$$
\phi: \mathcal{F} \rightarrow i_{*} \mathcal{F}_{\mid U} \rightarrow i_{*}\left(\mathcal{F}_{\mid U} / \mathcal{M}\right) .
$$

The kernel $\operatorname{ker}(\phi)$ is a $\mathcal{D}_{X}$-submodule of $\mathcal{F}$ and hence $\operatorname{ker}(\phi)$ equals either $\mathcal{F}$ or 0 . In particular, the restriction of $\operatorname{ker}(\phi)$ to $U$ is either $\mathcal{F}_{\mid U}$ or 0 . But, $\left.\operatorname{ker}(\phi)\right|_{U}$ equals $\mathcal{M}$. This completes the proof.

Lemma 3.5. Let $X$ be a variety over $k$ and let $\mathcal{F}$ denote a $\mathcal{D}_{X}$-module of finite length. Then the support of $\mathcal{F}$ is closed. 
Proof. As $\mathcal{F}$ has finite length, there exists a filtration

$$
0=\mathcal{F}_{0} \subseteq \mathcal{F}_{1} \subseteq \cdots \subseteq \mathcal{F}_{m}=\mathcal{F}
$$

by $\mathcal{D}_{X}$-submodules, such that the quotients $\mathcal{L}_{i}=\mathcal{F}_{i} / \mathcal{F}_{i-1}$ are simple $\mathcal{D}_{X}$-modules. Moreover, the support of $\mathcal{F}$ is the union of the supports of $\mathcal{L}_{i}, i=1, \ldots, m$. This reduces the statement to the case when $\mathcal{F}$ is a simple $\mathcal{D}_{X}$-module. So assume now that $\mathcal{F}$ is simple.

By Lemma 3.4 we may furthermore assume that $X$ is affine. Hence, there exists a global (nonzero) section $s$ of $\mathcal{F}$. By simplicity we must have $\mathcal{D}_{X} \cdot s=\mathcal{F}$, and hence the support of $\mathcal{F}$ coincides with the support of $s$ which is closed.

3.4. $F$-rationality and $\mathcal{D}$-modules. The concept of $F$-rationality comes from the theory of tight closure in commutative algebra. A local commutative ring in positive characteristic is called $F$-rational if every parameter ideal in $R$ is tightly closed. For the definition of tight closure see [9]. One may prove that every ideal in a strongly $F$-regular ring is tightly closed. In particular it follows that strongly $F$-regular rings are $F$-rational.

We quote the following crucial result by M. Blickle.

Theorem 3.1 (Corollary 4.10 in [2]). Let $R$ be regular, local, and F-finite. Let $A=R / I$ be a domain of codimension c. If $A$ is F-rational, then $H_{I}^{c}(R)$ is $D_{R^{-}}$ simple.

Here $F$-finite means that $R$ is finitely generated as a module over the subring $R^{p}$ of $p$-th powers. In particular, the theorem applies when $R$ is the localization of a finitely generated $k$-algebra. This leads to the following global result.

Proposition 3.2. Let $Y$ be an irreducible closed subvariety of codimension $c$ of a smooth variety $X$ over $k$. If all the local rings $\mathcal{O}_{Y, y}, y \in Y$, are $F$-rational, then the $\mathcal{D}_{X}$-module $\mathcal{H}_{Y}^{c}\left(\mathcal{O}_{X}\right)$ is simple.

Proof. By Lemma 3.3 there exists a finite length submodule $\mathcal{M}$ of $\mathcal{H}_{Y}^{c}\left(\mathcal{O}_{X}\right)$ such that the associated quotient $\mathcal{L}$ is a (nonzero) simple $\mathcal{D}_{X}$-module. Consider the corresponding short exact sequence:

$$
0 \rightarrow \mathcal{M} \rightarrow \mathcal{H}_{Y}^{c}\left(\mathcal{O}_{X}\right) \rightarrow \mathcal{L} \rightarrow 0 .
$$

The induced map of stalks

$$
0 \rightarrow \mathcal{M}_{x} \rightarrow \mathcal{H}_{Y}^{c}\left(\mathcal{O}_{X}\right)_{x} \rightarrow \mathcal{L}_{x} \rightarrow 0
$$

at a point $x$ in $X$, is then a short exact sequence of $\mathcal{D}_{X, x}$-modules. Using Theorem 3.1 and Lemma 3.2, we conclude that the middle term is a simple $\mathcal{D}_{X, x}$-module and hence that either $\mathcal{L}_{x}$ or $\mathcal{M}_{x}$ is zero. In particular, the support $\operatorname{Supp}\left(\mathcal{H}_{Y}^{c}\left(\mathcal{O}_{X}\right)\right)$, which is $Y$, is the disjoint union of the supports of $\mathcal{M}$ and $\mathcal{L}$. But, by Lemma 3.5. the supports of $\mathcal{L}$ and $\mathcal{M}$ are closed, and as $Y$ is irreducible, we conclude that either $\operatorname{Supp}(\mathcal{M})$ or $\operatorname{Supp}(\mathcal{L})$ is empty. Hence, $\mathcal{M}=0$ (as $\mathcal{L} \neq 0$ by assumption) and $\mathcal{H}_{Y}^{c}\left(\mathcal{O}_{X}\right)$ thereby coincides with the simple module $\mathcal{L}$.

\section{D-MOdules ON $G / B$}

Let $X$ denote a (generalized) flag variety $G / P$ and let $Y$ denote a Schubert variety in $X$ of codimension $c$. We assume that the characteristic of the ground field $k$ is positive. 
Theorem 4.1. The $\mathcal{D}_{X}$-module $\mathcal{H}_{Y}^{c}\left(\mathcal{O}_{X}\right)$ is simple.

Proof. By Proposition 3.2 it is enough to prove that the local rings $\mathcal{O}_{Y, y}, y \in Y$, are $F$-rational. But $Y$ is globally $F$-regular by Theorem 2.2 . In particular, the local rings $\mathcal{O}_{Y, y}$ are strongly $F$-regular and hence $F$-rational.

For a smooth algebraic variety $X$ with an action of an algebraic group $H$, there is a natural notion of an $H$-equivariant $\mathcal{D}_{X}$-module (see [10, p. 82).

Consider the category of $B$-equivariant holonomic $\mathcal{D}_{X}$-modules for $X=G / B$. Independently of $\operatorname{char}(k)$ a simple module in this category is uniquely given by its support, which is a Schubert variety (see [3], Theorem 4.6 for the positive characteristic case and [5], $\S 6.4$ for the characteristic zero case). We let $\mathcal{L}(w)$ denote the simple module with support $X(w)$ for $w \in W$. Independently of $\operatorname{char}(k)$ one may prove that $\mathcal{L}(w) \subseteq \mathcal{H}_{X(w)}^{c}\left(\mathcal{O}_{X}\right)$ with equality if $X(w)$ is smooth.

As a consequence of Theorem 4.1 we have the following result.

Theorem 4.2. Suppose that $X$ is over a field of positive characteristic. The unique simple $B$-equivariant and holonomic $\mathcal{D}_{X}$-module $\mathcal{L}(w)$ with support $X(w)$ is isomorphic to the local cohomology module

$$
\mathcal{H}_{X(w)}^{c}\left(\mathcal{O}_{X}\right)
$$

where $c$ denotes the codimension of $X(w)$.

In the next section we give an example showing that Theorem 4.2 does not hold in characteristic zero.

4.1. Nonsimplicity in characteristic zero. Consider the algebraic group $G=$ $\mathrm{SL}_{4}$ over the field $k$ with $\operatorname{char}(k)=0$ along with its 6-dimensional flag variety $X=G / B$. The Weyl group $W$ of $G$ is generated by the simple reflections $s_{1}, s_{2}, s_{3}$ numbered from left to right in the Dynkin diagram. We let $P_{v, w}$ denote the Kazhdan-Lusztig polynomial associated with $v, w \in W$ (cf. [12]).

Consider $w=s_{1} s_{2} s_{3} s_{2} s_{1}$. Then $X(w)$ is a codimension one Schubert variety in $X$. One may check that (in fact the singular locus of $X(w)$ is $X\left(s_{1} s_{3}\right)$ )

$$
P_{v, w}(q)= \begin{cases}1+q & \text { if } v \leq s_{1} s_{3}, \\ 1 & \text { if } v \not \leq s_{1} s_{3} .\end{cases}
$$

According to the Kazhdan-Lusztig conjecture (proved in [1, [5, [12]) we have the following formula for the simple module $\mathcal{L}(w)$ in the Grothendieck group of the category of $B$-equivariant and holonomic $\mathcal{D}$-modules on $X$ :

$$
[\mathcal{L}(w)]=\sum_{v \leq w}(-1)^{\ell(w)-\ell(v)} P_{v, w}(1)\left[\mathcal{H}_{C(v)}^{6-l(v)}\left(\mathcal{O}_{X}\right)\right] .
$$

The Schubert variety $X(w)$ is a local complete intersection since it has codimension one. Therefore $\mathcal{H}_{X(w)}^{j}\left(\mathcal{O}_{X}\right)$ is nonvanishing if and only if $j=1$. It follows by [1] (see 4.2 ) that we have an exact sequence

$$
0 \rightarrow \mathcal{H}_{X(w)}^{1}\left(\mathcal{O}_{X}\right) \rightarrow \mathcal{H}_{C(w)}^{1}\left(\mathcal{O}_{X}\right) \rightarrow \bigoplus_{v \leq w, \ell(w)-\ell(v)=1} \mathcal{H}_{C(v)}^{2}\left(\mathcal{O}_{X}\right) \rightarrow \ldots
$$


From this we get the formula

$$
\begin{aligned}
& {\left[\mathcal{H}_{X(w)}^{1}\left(\mathcal{O}_{X}\right)\right]=\sum_{v \leq w}(-1)^{\ell(v)-\ell(w)}\left[\mathcal{H}_{C(v)}^{6-\ell(v)}\left(\mathcal{O}_{X}\right)\right]} \\
& \quad=[\mathcal{L}(w)]+\sum_{v<w}(-1)^{\ell(v)-5}\left(1-P_{v, w}(1)\right)\left[\mathcal{H}_{C(v)}^{6-\ell(v)}\left(\mathcal{O}_{X}\right)\right] \\
& \quad=[\mathcal{L}(w)]+\sum_{v \leq s_{1} s_{3}}(-1)^{\ell(v)-2}\left[\mathcal{H}_{C(v)}^{6-\ell(v)}\left(\mathcal{O}_{X}\right)\right] \\
& \quad=[\mathcal{L}(w)]+\left[\mathcal{L}\left(s_{1} s_{3}\right)\right]
\end{aligned}
$$

in the Grothendieck group. It follows that $\mathcal{H}_{X(w)}^{1}\left(\mathcal{O}_{X}\right)$ is not a simple $\mathcal{D}_{X}$-module.

4.2. Decomposition of dual Verma modules. In this section we consider the category of $B$-equivariant and (filtration) holonomic $\mathcal{D}_{X}$-modules on $X=G / B$ over a field of positive characteristic. Associated to the $B$-orbit $C(w) \subset G / B$ we have the local cohomology sheaf $\mathcal{M}(w)=\mathcal{H}_{C(w)}^{c}\left(\mathcal{O}_{X}\right)$, where $c$ denotes the codimension of $C(w)$. The simple module $\mathcal{L}(w)=\mathcal{H}_{X(w)}^{c}\left(\mathcal{O}_{X}\right)$ admits a natural injection into $\mathcal{M}(w)$. Over a field of characteristic zero the decomposition of $\mathcal{M}(w)$ into simple modules ultimately rests on deep properties of intersection cohomology. The situation in positive characteristic is quite different. Applying the functor $\underline{\Gamma}_{X(w)}(-)$ to the local Grothendieck-Cousin complex for $\mathcal{O}_{X}$, we get a complex (see [11)

$$
0 \rightarrow \mathcal{L}(w) \rightarrow \mathcal{M}^{0} \rightarrow \mathcal{M}^{1} \rightarrow \cdots \rightarrow \mathcal{M}^{i} \rightarrow \cdots
$$

where

$$
\mathcal{M}^{i}=\bigoplus_{y \leq w, \ell(y)=\ell(w)-i} \mathcal{M}(y)
$$

and $\mathcal{L}(w)$ sits in degree $c-1$. The complex $\mathcal{M}^{\bullet}$ in (1) computes the cohomology $\mathcal{H}_{X(w)}^{j}\left(\mathcal{O}_{X}\right)$. Since $X(w)$ is Cohen-Macaulay, it follows by ([17, Chapitre III, Proposition (4.1)) that (1) is exact. This shows that we have the formula

$$
[\mathcal{L}(w)]=\sum_{y \leq w}(-1)^{\ell(w)-\ell(y)}[\mathcal{M}(y)]
$$

in the Grothendieck group of holonomic and $B$-equivariant $\mathcal{D}$-modules on $G / B$ in positive characteristic. Using Verma's identity (cf. [12, Remarks 3.3(b))

$$
\sum_{x \leq z \leq y}(-1)^{\ell(x)}(-1)^{\ell(z)}=\delta_{x, y}
$$

for all $x \leq y$ in $W$, one can invert (2) to get the decomposition

$$
[\mathcal{M}(w)]=\sum_{y \leq w}[\mathcal{L}(y)]
$$

for the "dual Verma module" $\mathcal{M}(w)$. This shows that the decomposition of $\mathcal{M}(w)$ into simple modules is multiplicity free and that $[\mathcal{M}(w): \mathcal{L}(y)]=1$ if $y \leq w$ and 0 otherwise. In the characteristic zero setting this decomposition is given by the value of inverse Kazhdan-Lusztig polynomials at 1 . The authors are unaware of 
any natural correspondence between the categories of holonomic and equivariant $\mathcal{D}$ modules in zero and positive characteristic even though they share many properties (e.g., simple modules and dual Verma modules parametrized by Schubert varieties).

\section{ACKNOWLEDGMENTS}

We are grateful to M. Kashiwara and V. B. Mehta for discussions related to this work. We also thank the referee for pointing out the connection to the RiemannHilbert correspondence in positive characteristic by Emerton and Kisin [8] in proving simplicity of local cohomology.

\section{REFERENCES}

1. A. Beilinson and J. Bernstein, Localisation de g-modules, C. R. Acad. Sci. Paris 292(1981), 15-18. MR0610137 (82k:14015)

2. Manuel Blickle, The intersection homology D-module in finite characteristic, Math. Ann. $\mathbf{3 2 8}$ (2004), 425-450. MR2036330 (2005a:14005)

3. R. Bögvad, Some results on D-modules on Borel varieties, J. Algebra 173 (1995), 638-667. MR:1327873 (97a:14015)

4. Homology Homotopy Appl. 4 (2002), 83-116. MR.1918185 (2003h:14030)

5. J. L. Brylinski and M. Kashiwara, Kazhdan-Lusztig conjecture and holonomic systems, Invent. Math. 64 (1981), 387-410. MR0632980 (83e:22020)

6. A. Grothendieck, EGA IV, Étude locale des schemas et des morphismes de schemas, Inst. Hautes Études Sci. Publ. Math. 32 (1967). MR0238860 (39:220)

7. _ Local Cohomology, Springer-Verlag, 1966. MR0224620 (37:219)

8. M. Emerton and M. Kisin, A Riemann-Hilbert correspondence for unit F-crystals, Astérisque 293 (2004). MR2071510(2005e:14027)

9. M. Hochster and C. Huneke, Tight closure and strong F-regularity, Mémoires de la Société Mathématique de France 38 (1989), 119-133. MR1044348 (91i:13025)

10. M. Kashiwara, Representation theory and D-modules on flag varieties, Astérisque 173-174 (1989), 55-109. MR1021510 (90k:17029)

11. M. Kashiwara and N. Lauritzen, Local cohomology and $\mathcal{D}$-affinity in positive characteristic, C. R. Acad. Sci. Paris 335 (2002), 993-996. MR1955575 (2004a:14020)

12. D. Kazhdan and G. Lusztig, Representations of Coxeter groups and Hecke algebras, Invent. Math. 53 (1979), 165-184. MR0560412 (81j:20066)

13. V. Lakshmibai and P. Magyar, Degeneracy schemes and Schubert varieties, Int. Math. Res. Notices 12 (1998), 627-640. MR1635873 (99g:14065)

14. N. Lauritzen and J. F. Thomsen, Line bundles on Bott-Samelson varieties, J. Alg. Geom. 13 (2004), 461-473. MR2047677(2005b:14087)

15. V. Mehta and A. Ramanathan, Frobenius splitting and cohomology vanishing for Schubert varieties, Ann. Math. 122 (1985), 27-40. MR0799251 (86k:14038)

16. A. Ramanathan, Equations defining Schubert varieties and Frobenius splitting of diagonals, Pub. Math. IHES 65 (1987), 61-90. MR0908216 (88k:14032)

17. C. Peskine, L. Szpiro, Dimension projective finie et cohomologie locale, Pub. Math. IHES 42 (1973), 47-119. MR0374130 (51:10330)

18. K. E. Smith, Globally F-regular varieties: Applications to vanishing theorems for quotients of Fano varieties, Mich. J. Math. 48 (2000), 553-572. MR.1786505(2001k:13007)

19. T. Springer, Linear Algebraic Groups, Springer Verlag (2nd edition), 1998. MR1642713 (99h:20075) 
Institut for matematiske fag, Aarhus Universitet, Ny Munkegade, DK-8000 Arhus, C Denmark

E-mail address: niels@imf.au.dk

Institut for matematiske fag, Aarhus Universitet, Ny Munkegade, DK-8000 Arhus, C DENMARK

E-mail address: ab061278@imf.au.dk

Institut for matematiske fag, Aarhus Universitet, Ny Munkegade, DK-8000 Arhus, C DENMARK

E-mail address: funch@imf.au.dk 